
cultures

Les cahiers de l'Acedle

6-1 | 2009

L'Alsace au cœur du plurilinguisme (2)

\title{
La motivation pour la langue étrangère à l'école primaire : apprentissage en autonomie à l'ordinateur
}

Shona Whyte

\section{OpenEdition}

1 Journals

Édition électronique

URL : http://journals.openedition.org/rdlc/2223

DOI : $10.4000 /$ rdlc. 2223

ISSN : 1958-5772

Éditeur

ACEDLE

Référence électronique

Shona Whyte, "La motivation pour la langue étrangère à l'école primaire : apprentissage en autonomie à l'ordinateur », Recherches en didactique des langues et des cultures [En ligne], 6-1 | 2009, mis en ligne le 01 avril 2009, consulté le 24 avril 2019. URL : http://journals.openedition.org/rdlc/2223 ; DOI : $10.4000 /$ rdlc.2223

Ce document a été généré automatiquement le 24 avril 2019.

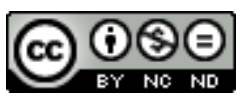

Recherches en didactique des langues et des cultures is licensed under a Creative Commons AttributionNonCommercial-NoDerivatives 4.0 International License 


\title{
La motivation pour la langue étrangère à l'école primaire : apprentissage en autonomie à l'ordinateur
}

\author{
Shona Whyte
}

\section{Introduction}

\subsection{Acquisition des langues secondes et socioconstructivisme}

1 Toute tentative de retracer l'histoire des recherches en acquisition des langues secondes (ALS) mène naturellement à distinguer trois grandes phases: la période comportementaliste, où l'on considère l'apprenant comme simple objet déterminé par les habitudes créées par son environnement et dépourvu d'initiative intellectuelle ; l'ère du cognitivisme, pendant laquelle la métaphore dominante est l'apprenant comme ordinateur, qui reçoit des données (input) et en produit d'autres (output) suite à l'intervention d'un certain nombre de sous-modules contrôlant différents aspects de la réception et la production de langage; et nous entrons plus récemment dans une phase socio-cognitive ou constructiviste, où l'on s'intéresse davantage au contexte social dans laquelle l'acquisition a lieu (voir par exemple le résumé récent de Johnson, 2004).

2 Firth et Wagner (Firth \& Wagner, 1997) ont été parmi les premiers à formuler une opposition raisonnée à ce qu'ils considèrent comme la dérive cognitiviste, dénonçant des approches étroitement linguistiques basées sur des conceptions réductrices des notions disciplinaires fondamentales telles que locuteur natif ou encore interlangue, et appellent à une démarche plus holistique qui chercherait à :

understand and explicate how language is used as it is being acquired through interaction, and used resourcefully, contingently, and contextually (Firth \& Wagner 2007: 768, emphase originale). 
Cet appel pour l'intégration d'une dimension sociale au plan cognitif ayant été largement entendu (pour en juger il suffit de noter le numéro spécial du Modern Language Journal de 2007 qui re-publie l'article original de Firth et Wagner et se consacre à l'étude de ses effets sur les recherches récentes, Sieloff-Magnan, 2007), le paradigme sociocognitif prend désormais toute sa place dans les recherches sur l'acquisition.

4 Il en va de même dans le domaine de la psychologie de l'éducation, où la notion $\mathrm{d}^{\prime}$ agentivité personnelle ancrée dans des structures sociales développée par Albert Bandura (voir par exemple Bandura, 2001) a contribué à une orientation des recherches vers les effets de la motivation des individus et des groupes sur la réussite dans des tâches. L'auteur insiste sur le concept d'auto-efficacité ou du sentiment d'efficacité personnelle, qu'il définit comme la confiance que ressent un individu en sa capacité à exercer une certaine mesure de contrôle sur son propre fonctionnement et sur les évènements qui surviennent dans son environnement (Bandura, 2001 : 10). L'auteur met le sentiment d'auto-efficacité au premier plan de la théorie sociocognitive, considérant que ce dernier joue un rôle central dans la régulation de la motivation, influant sur les choix d'actions d'un individu, sur sa persistance face aux obstacles, et sur la quantité d'effort fourni lors de l'accomplissement d'une tâche (Manolopoulou-Sergi, 2004).

\subsection{Motivation et autonomie}

D'après Ehrman et al. (Ehrman \& al., 2003), les apprenants de langues secondes les plus motivés et ceux qui rencontrent le plus de succès font preuve d'un sentiment d'efficacité personnelle prononcé. Ils sont convaincus qu'ils exercent un contrôle significatif sur les résultats de leurs apprentissages, montrent une attitude positive à l'apprentissage en général, et affichent à la fois la volonté de s'intégrer dans le groupe (ex. la classe) et de travailler de manière autonome (Ehrman \& al., 2003 : 321). Au concept de l'efficacité de l'individu et de son estimation de sa probabilité de réussite lors d'une tâche (Cotterall, 1999) s'ajoute la notion de la valeur accordée à cette tâche par l'individu, élément central du modèle "expectancy-value" de Eccles et Wigfield (Wigfield \& Eccles, 2000). Au sein de l'approche des buts de l'accomplissement (achievement goal theory), le but de maitrise est distingué de celui de la performance. Le premier concerne l'individu qui cherche à développer une certaine compétence par intérêt pour celle-ci, alors que le deuxième caractérise un apprenant qui veut démontrer ses compétences aux autres, ou éviter de révéler ses lacunes (Liem \& al, à paraître).

6 L'intégration dans le domaine de l'ALS de ces recherches en psychologie de l'éducation au travaux plus traditionnels en psychologie sociale (Gardner \& Lambert, 1972; Gardner, 1985) a permis de dépasser les oppositions entre motivation intégrative et motivation instrumentale qui dominaient la recherche sur la en motivation pour l'apprentissage d'une langue seconde mais qui ne permettaient guère de cerner tous les éléments touchant à ce domaine dans la classe de langue étrangère, comme le soulignent Dörnyei et Csizér (Dörnyei \& Csizér, 1998 : 204-5). Ces derniers proposent un modèle de motivation pour l'apprentissage d'une langue étrangère qui repose sur trois éléments: la langue, l'apprenant et la situation d'apprentissage. La partie langue comprend les motivations intégrative et instrumentale qui peuvent être ressenties, tandis que les notions d'autoefficacité, de besoin d'accomplissement sont le propre du deuxième volet, celui de l'apprenant. Enfin la troisième partie concerne la situation d'apprentissage et recouvre les 
notions telles que l'intérêt et la pertinence des tâches, la motivation affiliative (vers les pairs ou vers l'enseignant) et les normes de la classe.

7 L'étude de la motivation chez les jeunes apprenants en milieu scolaire permet de justifier de l'importance des volets apprenant et situation d'apprentissage dans la classification de Dörnyei et Csizér (Dörnyei \& Csizér, 1998; 2005). Lors de son analyse des travaux effectués dans ce domaine, Nikolov (Nikolov,1999) fait référence à l'une des premières grandes expériences de l'apprentissage précoce à l'école, celle de l'enseignement du français langue étrangère en primaire en Angleterre dans les années soixante-dix. D'après l'auteur, les travaux de Burstall (Burstall, 1980) démontrent un lien entre la dimension des écoles et la réussite des élèves : le niveau linguistique des élèves dans de petites écoles en milieu rural dépasse systématiquement celui de leurs camarades dans de grands établissements urbains :

This surprising result is explained by the author by the fact that pupils in small schools tend to form closer relationships with their teachers, and this early establishment of good teacher-pupil relationships can explain the higher level of achievement in French and the subsequent development of positive attitudes towards further learning (Nikolov, 1999: 36).

Lors de ses propres recherches longitudinales approfondies sur la motivation des élèves hongrois en classe d'anglais à l'école primaire (l'auteur suit trois cohortes de 15 élèves sur huit ans), Nikolov retrouve la même motivation affiliative ainsi que l'importance de la situation d'apprentissage :

the most important motivating factors for children between 6 and 14 years of age included positive attitudes towards the learning context and the teacher: intrinsically motivating activities, tasks and materials; and they were more motivated by classroom practices than integrative or instrumental reasons (Nikolov, 1999: 53).

L'auteur note pourtant une évolution au cours du parcours scolaire, traçant le développement du but de maitrise qui prend progressivement le pas sur les facteurs externes tels que les récompenses ou l'approbation (de l'enseignant): "Knowledge as an aim gradually overtook the role of external motivating factors like rewards and approval" (Nikolov, 1999 : 53).

10 Si la motivation des enfants se situe en général dans le hic et nunc de la classe de langue, l'apprenant adulte, lui, peut faire preuve d'une plus grande autonomie. Plusieurs études sont consacrées aux capacités de l'apprenant adulte à travailler de manière autonome sur la langue étrangère. Mills et al. (Mills \& al. 2007) comparent le niveau linguistique d'étudiants débutants en français langue étrangère aux États-Unis avec leur autoévaluation de leur sentiment d'efficacité personnelle pour l'apprentissage des langues et d'autres variables motivationnelles. Les auteurs rendent compte de corrélations significatives entre la réussite linguistique, d'une part, et le sentiment d'efficacité personnelle (self-efficacy) et l'estimation de la valeur de la langue française (perceived value of language and culture), d'autre part. Cette dernière variable recouvre la perception par l'apprenant de l'importance, de l'intérêt et de l'appréciation des connaissances de la langue et de la culture françaises (Mills \& al., 2007 : 424). Ainsi les étudiants qui font preuve du plus grand intérêt pour la langue et qui sont les plus convaincus de leur propre capacité à l'apprendre sont en effet ceux qui réussissent le mieux. 
11 Si l'étude de Mills et al. (2007) tend à suggérer qu'une motivation positive facilite l'acquisition, d'autres recherches montrent le revers de la médaille: une absence de motivation rend tout apprentissage difficile. Lors d'une étude de la motivation pour l'apprentissage de l'anglais parmi les étudiants d'une université hongkongaise, Spratt et al. (2002) mettent en cause le principe de l'apprentissage autonome comme moteur de la motivation et ensuite de la réussite. Au travers de questionnaires et d'entretiens avec des étudiants, ces auteurs révèlent un manque de motivation qui empêche les apprenants de chercher des occasions d'utiliser la langue étrangère. Pour ces auteurs, il serait utile de stimuler la motivation chez les apprenants avant de chercher à leur inculquer des stratégies pour un travail autonome (Spratt \& al., $2002: 263$ ).

\subsection{Apprentissage médié par ordinateur}

La notion d'autonomie dans l'apprentissage se retrouve ainsi au premier plan dans de nombreux domaines de recherche touchant à la situation d'apprentissage qui nous intéresse dans ce travail. Pour les socioconstructivistes, les connaissances sont construites par l'individu selon ses propres expériences ; la situation d'apprentissage doit obligatoirement lui permettre de comprendre à sa façon à partir de son propre ressenti (Jonassen \& al, 1995). Kennewell et Morgan (2006) ont cherché à promouvoir cette autonomie dans l'apprentissage par le jeu. Dans leurs travaux sur l'apprentissage de l'utilisation de logiciels en situation non-dirigée, ils valorisent, suivant Bandura, l'expérience de maîtrise (mastery experience) comme source primordiale d'information pour l'apprenant sur son efficacité personnelle. Ils démontrent comment cette expérience peut même être mise à la portée des enfants de 7 ans, qui, lors des séances de familiarisation avec un outil informatique menées en centre de vacances :

were highly engaged in activity and gained a worthwhile number of skills within a short period in an unstructured learning environment with no external goals (Kennewell \& Morgan, 2006: 277).

13 L'autonomie de l'apprenant prend toute son importance dans le domaine de l'apprentissage des langues assisté par ordinateur (ALAO / CALL), car les nouvelles technologies poussent l'apprenant à travailler seul, et celui qui les maitrise reçoit une alphabétisation électronique qui lui ouvre un potentiel pour apprendre tout au long de la vie (Blin, 2004). Pour l'enseignant, la possibilité de transférer la responsabilité du choix de ressources et des stratégies d'apprentissage sur l'apprenant à travers les ressources informatiques devient ainsi un impératif didactique (Chapelle, 1998; Figura \& Jarvis, 2007).

14 Pour toutes ces raisons, l'apprentissage d'une langue étrangère à partir de supports informatiques revêt ainsi une importance à la fois théorique et pratique. Des modèles intégrant l'environnement de l'ordinateur aux processus d'acquisition sont proposés par Simina et Hamel (2005) et Bertin et Narcy-Combes (2007). Ces derniers rappellent la nécessité d'examiner les capacités technologiques à la lumière de la théorie de l'ALS et l'importance cruciale de la théorie pour guider même les études les plus empiriques (Bertin \& Narcy-Combes, 2007 : 444). Simina et Hamel (2005) situent à l'intersection du cadre socioconstructiviste et de l'environnement ALAO un contexte d'apprentissage idéalisé :

... it is learner-centred, meaning that the learner is free to make his or her own interpretations. It also promotes authenticity through context-rich and 
experience-based activities which enables learners to associate new with prior knowledge. Moreover social interaction is crucial for the sharing of multiple representations, reflection and monitoring ... Finally it embeds scaffolding for the manipulation of the learner's attention to form and meaning and for collaboration to achieve the construction of knowledge (Simina \& Hamel, 2005: 224).

Des recherches empiriques étayent ces propos. En ce qui concerne la richesse et l'authenticité de l'input, Ramirez Verdugo et Alonso Belmonte (2007) démontrent l'intérêt des sites Internet pour enfants anglophones pour l'apprentissage de l'anglais langue étrangère en Espagne. Les auteurs mènent une étude sur l'utilisation des contes en ligne trouvés sur Kindersite, réalisée auprès de quelques 200 écoliers âgés de 6 ans sur une période de 5 mois durant le temps scolaire et relèvent un (léger) effet positif sur la compréhension de la langue parlée pour le groupe test par rapport à un groupe contrôle qui a subi des méthodes traditionnelles sans ordinateur.

Le potentiel social d'un travail collaboratif par ordinateur a fait l'objet de deux études axées sur l'enseignement de l'anglais à l'école en France. Pour Grosbois (2007), la création de supports multimédia pour les élèves est l'occasion d'étudier l'interaction entre enseignants ; elle estime qu'une ressource Internet " composée de textes, d'images, et de sons, traitant de sujets présentant un intérêt certain pour les enfants [peut] favoriser l'éveil linguistique et culturel d'écoliers en primaire " (Grosbois, 2007 : 105). D'autre part, lors d'une étude sur l'utilisation de cyberquêtes sur le monde anglophone par des élèves de cycle 3, Catroux examine l'interaction des dyades sous un angle socioconstructiviste et conclut que cette activité " peut induire une prise d'autonomie chez des enfants jeunes " (Catroux, $2006: 67$ ).

Enfin, le potentiel d'étayage (scaffolding) qu'offre l'environnement numérique fait l'objet d'une étude sur l'acquisition lexicale par Jones (2004). S'appuyant sur des recherches montrant l'utilité d'associer de nouveaux lexèmes à des représentations visuelles, elle compare la reconnaissance et la production de nouveaux mots présentés avec et sans annotations audiovisuelles chez des étudiants débutants en français langue étrangère. L'auteur conclut que l'acquisition de vocabulaire est particulièrement soutenue quand les étudiants ont la possibilité de recourir à des images et à des annotations écrites, mais met en garde contre une trop grande richesse d'annotations, qui peut encombrer la mémoire et produire une surcharge cognitive (Jones, $2004: 134$ ).

\subsection{Le jeune apprenant et l'ordinateur en classe de langue étrangère}

$\mathrm{Au}$ cours de cette première partie de l'article, nous avons examiné à tour de rôle les facteurs différents qui influent sur notre sujet de recherche : le constructivisme en ALS, la motivation et l'autonomie des apprenants et les apports de l'ALAO. Il convient à présent de synthétiser les conclusions de ces travaux pour le cas du jeune apprenant devant l'ordinateur en classe de langue.

19 Le modèle socioconstructiviste nous encourage à considérer le jeune apprenant, tout comme l'adulte, comme acteur de son propre apprentissage, qui peut et doit endosser des responsabilités quant au choix des ressources et aux stratégies d'apprentissage (Félix, 2005). Nous pouvons lui proposer de choisir parmi des activités sur des sites Internet avec un contenu audio-visuel riche, et nous attendre à ce qu'une approche non dirigée, guidée uniquement par l'aspect ludique de l'activité, puisse conduire à des apprentissages réels (Kennewell \& Morgan, 2006). Nous anticipons que, en dehors de l'intérêt de cette activité, 
la motivation principale du jeune apprenant restera l'approbation de l'enseignant (Nikolov, 1999).

Une étude empirique exploratrice menée dans la classe de langue étrangère à l'école primaire nous permet d'examiner les interactions entre apprentissages médiés par l'ordinateur, motivation et autonomie chez le jeune apprenant.

\section{Méthode}

Nous examinons le cas de l'utilisation d'activités en ligne dans une petite classe multiniveaux d'anglais langue étrangère. Nous nous intéressons aux effets de cette activité sur :

- l'auto-évaluation des connaissances lexicales ;

- la capacité de participer en interaction orale ;

- la motivation pour la langue et pour l'outil informatique.

Les participants sont répartis entre un groupe test, qui travaille sur des jeux interactifs en anglais et un groupe contrôle, qui travaille sur une cyberquête en français ${ }^{1}$. Nous comparons les mesures des capacités linguistiques et des motivations des deux groupes, relevées avant et après l'intervention.

\subsection{Participants}

Les 18 élèves d'une classe entière de CE1, CE2, CM1 et CM2 (âgés de 7 à 11 ans) dans une petite école de village de l'arrière-pays niçois ont participé à une expérience menée durant 9 semaines pendant la dernière période de l'année scolaire (mai-juin 2007). Tous suivaient des cours d'anglais avec l'auteur en tant qu'intervenante extérieure depuis le début de l'année scolaire, dans un enseignement organisé par ateliers et axé sur l'exploitation de ressources pour l'apprentissage de l'anglais disponibles sur Internet.

Les douze garçons et six filles étaient repartis sur les quatre niveaux de la façon suivante : 3 élèves en CE1, 5 en CE2, 6 et CM1 et 4 en CM2. Les élèves de CE1 ayant débuté en anglais à la rentrée 2006 avaient suivi une trentaine d'heures de cours. La plupart des autres élèves avaient bénéficié d'un, de deux, voire de trois ans d'anglais au cours de leur scolarité.

\subsection{Matériel}

Les participants à l'expérience travaillaient en autonomie sur l'un des six ordinateurs de la classe en se servant d'activités en ligne proposées sur un site Internet expérimental créé par l'auteur à l'intention de la classe (Cherry English). Chaque élève devait travailler soit sur des jeux interactifs en anglais, soit sur une cyberquête sur les pays anglophones en français, au rythme de deux ou trois séances hebdomadaires de 10 minutes chacune, organisées par l'enseignante de la classe en dehors des séances d'anglais.

Les jeux interactifs sont réunis sur le site par thème et didactisés par l'auteur ; certains d'entre eux faisaient l'objet d'exploitations didactiques lors des séances d'anglais précédant ou pendant la période de l'expérimentation, mais une grande partie d'entre eux a été rajoutée pour l'étude. Les apprenants choisissent les activités librement à partir 
des rubriques du site, la contrainte étant de rester sur les sites indiqués et de noter les activités utilisées sur un cahier de bord.

Les activités de cyberquête ont été conçues par l'auteur à partir d'une activité de classe centrée sur l'envoi de cartes postales vers les pays anglophones: " Postcard Pete ". La cyberquête comprend seulement des sites francophones et permet de découvrir des aspects de la culture des différents pays anglophones. Les apprenants pouvaient choisir l'ordre dans lequel ils abordaient les différents pays, mais devaient visiter tous les sites, notant les réponses aux questions dans leur cahier de bord.

\subsection{Protocole et mesures}

Les élèves sont ainsi répartis en deux groupes : un groupe test, qui travaille sur les jeux interactifs en anglais, et un groupe contrôle, qui suit la cyberquête en français. Pour assurer l'équilibre des deux groupes quant au niveau et au sexe des élèves, les participants sont d'abord triés selon ces deux variables, puis affectés de manière aléatoire à l'un des groupes. Enfin, pour vérifier la similarité des groupes, le niveau d'autonomie de chaque élève est évalué indépendamment par l'auteur et l'enseignante de la classe.

\subsubsection{Autonomie}

Sur une échelle de 0 (faible) à 2 (fort) sur trois variables (niveau intellectuel, maîtrise de soi, capacité à travailler seul), la moyenne des notes des deux enseignantes présentée dans le tableau 1 montre qu'il n'y a pas de disparité entre les groupes: des apprenants très indépendants (avec un score maximum de 2 points) côtoient des apprenants moins autonomes (avec des scores de 0,33 ou 0,5 sur 2 points) dans les deux groupes.

Dans tous les tableaux de ce travail, les participants sont identifiés par leurs initiales et présentés par niveau scolaire et par groupe, les filles en italique.

Tableau 1 - Autonomie des élèves, notée sur 2 par les enseignantes.

\begin{tabular}{|c|c|c|c|c|}
\hline & \multicolumn{2}{|c|}{ TEST } & \multicolumn{2}{l|}{ CONTROLE } \\
\hline CE1 & DM & 0,5 & BP & 1,5 \\
\hline & & & $S T$ & 0,67 \\
\hline & & & & \\
\hline CE2 & EM & 2 & KT & 1 \\
\hline & ET & 1,5 & NC & 0,33 \\
\hline & SN & 0,67 & & \\
\hline & & & & \\
\hline CM1 & DY & 1,33 & CA & 1,5 \\
\hline & KY & 1,33 & MX & 1,67 \\
\hline
\end{tabular}




\begin{tabular}{|c|c|c|c|c|}
\hline & TE & 1,8 & RA & 2 \\
\hline & & & & \\
\hline CM2 & CE & 1,67 & $\mathrm{AX}$ & 1 \\
\hline & MO & 2 & $\mathrm{GM}$ & 1,67 \\
\hline Moyenne & & 0,44 & & 0,44 \\
\hline
\end{tabular}

\subsubsection{Auto-évaluation des connaissances lexicales}

Une auto-évaluation des connaissances lexicales est menée avant et après l'intervention. À partir d'une fiche de cinquante images représentant des lexèmes de domaines étudiés en classe (ex. couleurs, animaux, météo) et triés selon leur fréquence (souvent, rarement ou jamais vu en classe), l'élève entoure les mots qu'il croit pouvoir restituer en anglais (et qui lui seront demandés lors d'un entretien oral). Sont comptabilisés les mots entourés après déduction d'éventuelles erreurs lors de l'entretien.

Trois fiches comparables sont préparées selon les mêmes critères (domaines lexicaux, fréquence) pour éviter un effet de répétition lors du post-test; le score présenté représente la différence entre pré- et post-tests.

\subsubsection{Capacité à participer en interaction orale}

L'élève participe avant et après l'intervention à un entretien individuel avec l'auteur de trois à cinq minutes comprenant dix questions, tirées des interactions habituelles de la classe (prénom, date, météo) ou des images de la fiche (ce que l'apprenant aime, possède : I like, I've got). Les entretiens sont enregistrés et transcrits, puis les réponses des élèves sont analysées pour déterminer le niveau de compréhension de l'apprenant ainsi que sa capacité à s'exprimer à l'oral.

Les réponses sont codées de 0 (absence de réponse) à 3 (très bonne réponse) selon leur niveau de complétude; sont prises en compte la promptitude des réponses en ce qui concerne la compréhension et la richesse de la production dans le cas de l'expression orale. Les critères de codage se trouvent dans l'annexe 1. Comme pour les connaissances lexicales, les scores rapportés représentent la différence des réponses données en pré- et post-tests.

\subsubsection{Motivation pour la langue et pour l'informatique}

Les motivations des apprenants sont jaugées selon trois mesures :

- un questionnaire de motivation générale: réponses écrites sur un questionnaire comprenant 5 questions ouvertes;

- un questionnaire de motivation pour l'apprentissage à l'ordinateur : évaluation de jeux interactifs spécifiques sur une échelle de 4 ;

- les notations sur le cahier de bord : indications du temps passé en autonomie aux activités à l'ordinateur écrites par chaque participant.

Le questionnaire de motivation générale (voir annexe 2) cible l'opposition entre intrinsèque et extrinsèque, les facteurs apprenant tels que l'intérêt et le sentiment 
d'efficacité personnelle, ainsi que les éléments de la situation d'apprentissage (activités, enseignant), s'appuyant sur Dörnyei et Csizér (1998) et Nikolov (1999). Ce questionnaire est complété avant et après l'intervention, comme c'est le cas pour un deuxième questionnaire sur les activités spécifiques en ligne qui sont travaillées lors de la période écoulée.

Le questionnaire sur les jeux spécifiques consiste en un tableau à double entrée qui permet à l'apprenant de noter son opinion de chaque jeu sur une échelle de quatre (émoticônes pour très bien, bien, $\mathrm{OK}$, et pas bien du tout); il est complété devant l'ordinateur, selon la méthode de Read et al. (2002). Enfin, un complément d'information sur l'utilisation du site Cherry English par les apprenants en dehors du temps scolaire est sollicité oralement par l'auteur et noté sur ce questionnaire.

Le troisième instrument pour mesurer la motivation est le cahier de bord, cahier individuel utilisé par chaque apprenant pour noter la date et l'heure de chaque séance en autonomie sur l'ordinateur, ainsi que le thème abordé. Ces informations permettent de calculer le nombre de passages à l'ordinateur ainsi que la durée totale de l'intervention.

\subsection{Résultats}

Nous présentons d'abord les résultats des analyses linguistiques, regroupant l'autoévaluation des connaissances lexicales et la capacité à interagir oralement dans un premier temps.

\subsubsection{Connaissances lexicales et capacité à interagir oralement}

Le tableau 2 résume les résultats des tests de connaissances lexicales et d'interactions présentées en sections 2.3.2 et 2.3.3. L'évolution entre pré- et post-test est présentée pour chaque participant pour le groupe test et le groupe contrôle. Pour DM, par exemple, élève de CE1 dans le groupe test, le niveau en lexique, mesuré au nombre d'images identifiées en anglais, progresse de $120 \%$, tandis que le progrès en interaction est de 1 point pour la compréhension et de 0,3 point pour l'expression sur une échelle de 3 .

Les scores considérés comme indiquant un progrès net sont indiqués en vert, une évolution moyenne en jaune, et des progrès plus discrets en rouge.

Tableau 2 - Différences de scores entre pré- et post-tests.

\begin{tabular}{|c|c|c|c|c|c|c|c|c|}
\hline & \multicolumn{3}{|c|}{ TEST } & \multicolumn{4}{c|}{ CONTROLE } \\
\hline & & Lexique & \multicolumn{2}{|c|}{ Interaction } & & Lexique & \multicolumn{2}{|c|}{ Interaction } \\
\hline & & & COMP & EXP & & & COMP & EXP \\
\hline CE1 & DM & $120 \%$ & 1 & 0,3 & BP & $112 \%$ & 0,3 & 0,8 \\
\hline & & & & & $S T$ & $-11 \%$ & 1,3 & 0 \\
\hline & & & & & & & & \\
\hline CE2 & EM & $37 \%$ & 1,1 & 0,9 & $K T$ & $5 \%$ & 1,1 & 1 \\
\hline
\end{tabular}




\begin{tabular}{|c|c|c|c|c|c|c|c|c|}
\hline & ET & $108 \%$ & 1 & 0,4 & $\mathrm{NC}$ & $-31 \%$ & 0,3 & 0 \\
\hline & $\mathrm{SN}$ & $13 \%$ & 0,2 & 0,3 & & & & \\
\hline CM1 & DY & $11 \%$ & $-0,2$ & $-0,1$ & $\mathrm{CA}$ & $92 \%$ & 0,2 & 0,3 \\
\hline & $\mathrm{KY}$ & $26 \%$ & 0,7 & 0,4 & $\mathrm{MX}$ & $25 \%$ & 0,7 & $-0,5$ \\
\hline & $\mathrm{TE}$ & $28 \%$ & 0,2 & $-0,4$ & $\mathrm{RA}$ & $14 \%$ & 1,6 & 1 \\
\hline & & & & & & & & \\
\hline CM2 & $\mathrm{CE}$ & $0 \%$ & 0 & $-0,4$ & $\mathrm{AX}$ & $-9 \%$ & 0,6 & 0 \\
\hline & $\mathrm{MO}$ & $14 \%$ & 0,2 & $-0,2$ & $\mathrm{GM}$ & $48 \%$ & $-0,3$ & 0,5 \\
\hline & & $40 \%$ & 0,47 & 0,13 & & $27 \%$ & 0,64 & 0,34 \\
\hline
\end{tabular}

LEXIQUE : différence entre nombre d'images identifiées

INTERACTION : différence de moyennes sur 3 en compréhension et expression mesures différentes. Mais, dans le groupe test l'évolution positive est plus nette: huit élèves sur neuf ont des scores plus élevés en reconnaissance lexicale (la neuvième restant au même niveau), alors que le groupe contrôle montre plus de variabilité (trois apprenants reculent). Les apprenants du groupe test qui progressent le font de façon plus globale : trois d'entre eux, DM, EM ET obtiennent des scores nettement plus élevés à la fois en lexique et en interaction, deux autres $\mathrm{SN}$ et KY montrent des progrès moins nets mais également répartis sur les deux mesures. Dans le groupe contrôle, en revanche, quatre apprenants seulement progressent, dont deux, BP et RA, sur les deux mesures, et ce de façon moins marquée que dans le groupe test.

Les résultats ne permettent pas de déceler un effet significatif sur la reconnaissance des mots ni sur la capacité à participer en interaction orale grâce au travail en autonomie sur les jeux interactifs à l'ordinateur. Cependant, ils fournissent quelques pistes de réflexion pour l'interprétation des motivations des apprenants.

\subsubsection{Motivation pour la langue et pour l'informatique}


- succès de l'apprenant (+ parce que j'arrive bien, - j'arrive pas trop) ;

- appréciation des activités (+ parce qu'on fait plein de choses différentes) ;

- appréciation de l'enseignante (+ parce que Shona est gentille);

- appréciation de l'ordinateur (+ parce qu'on va à l'ordinateur).

Après analyse des questionnaires, trois profils distincts se dégagent, indiqués au tableau 3 par trois couleurs différentes.

Tableau 3 - Facteurs motivationnels pour chaque participant avant (1) et après (2) l'intervention.

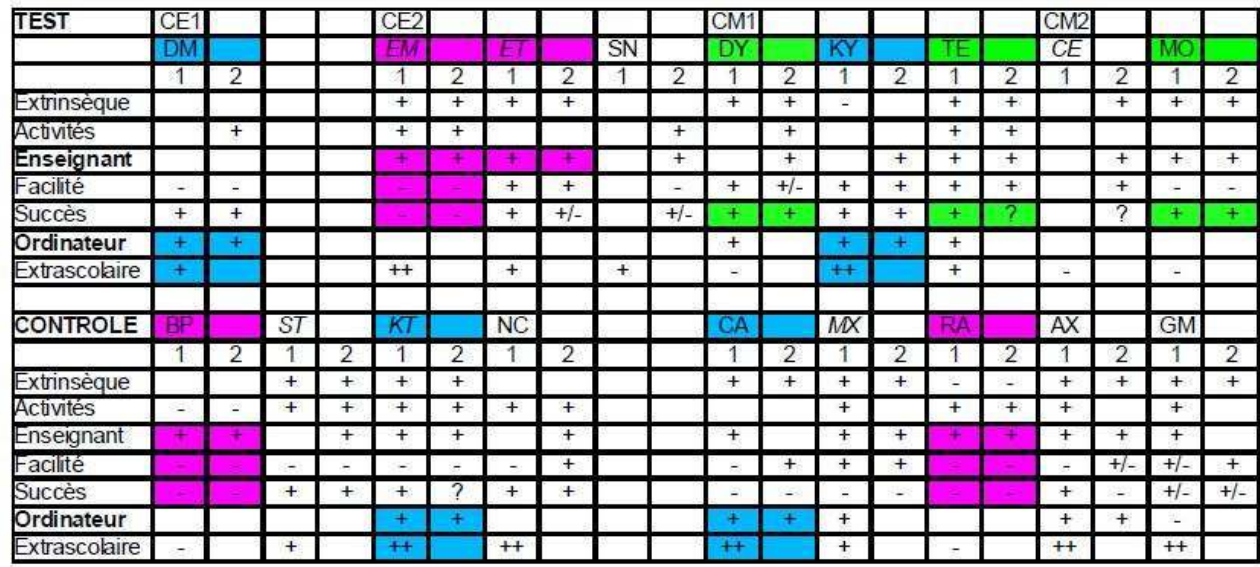

Pour quatre apprenants, marqués en bleu, DM, KY, KT et CA, c'est la motivation de l'ordinateur qui prime. Pour tous, l'atelier ordinateur du cours d'anglais est le préféré (avant le travail en autonomie en groupe et le travail avec l'intervenante). Tous ont accès à Internet à la maison, et trois sur quatre affirment déjà y avoir visité le site Cherry English . Deux autres apprenants, AX et GM, prétendent également utiliser le site en dehors du temps scolaire sans pour autant présenter un progrès en anglais; nous supputons un effet de plafond dans les tests qui sera discuté plus loin.

Un deuxième profil apprenant concerne la relation avec l'enseignante et le sentiment d'efficacité personnelle. Quatre autres apprenants, indiqués en rose, EM, ET, BP et RA, préfèrent l'atelier avec l'enseignante lors du cours d'anglais, mais jugent leur propre performance de façon négative : pour trois d'entre eux, la langue anglaise est difficile et ils ne sont pas satisfaits de leurs capacités à la comprendre et à l'utiliser.

Enfin, un troisième groupe d'apprenants, marqué en vert, semble bénéficier d'un meilleur sentiment d'efficacité personnelle ; il s'agit de DY, TE, et MO, trois membres plus âgés du groupe test. Nous pouvons imaginer que le sentiment de maîtrise de la matière de ces apprenants exerce un effet négatif sur leur volonté de fournir un effort. Les propos de MO sur le questionnaire de motivation générale le confirment : à la question : "penses-tu que tu arrives bien en anglais ? Pourquoi ?" il répond : "oui, parce que Shona m'interroge jamais" 2 .

En effet, tous les élèves de CM1 et de CM2, à l'exception de MX et RA, obtiennent un score de plus de 2 sur 3 en compréhension au moment du pré-test, et tous y parviennent lors du post-test, suggérant un effet de plafond sur cette mesure. Le tableau 4 donne les scores des pré- et post-tests pour la compréhension et l'expression orales. 
Tableau 4 - Scores en compréhension et expression orales avant (1) et après (2) l'intervention.

\begin{tabular}{|c|c|c|c|c|c|c|c|c|}
\hline & \multicolumn{4}{|c|}{ TEST } & \multicolumn{4}{|c|}{ CONTROLE } \\
\hline & & & Compréhension & Expression & & & Compréhension & Expression \\
\hline CE1 & DM & 1 & 0,2 & 0,6 & $\mathrm{BP}$ & 1 & 1,6 & 0,6 \\
\hline & & 2 & 1,2 & 0,9 & & 2 & 1,9 & 1,4 \\
\hline & & & 1 & 0,3 & & & 0,3 & 0,8 \\
\hline & & & & & $S T$ & 1 & 0,7 & 1 \\
\hline & & & & & & 2 & 2 & 1 \\
\hline & & & & & & & 1,3 & 0 \\
\hline CE2 & EM & 1 & 1,2 & 0,7 & $K T$ & 1 & 1,2 & 1 \\
\hline & & 2 & 2,3 & 1,6 & & 2 & 2,3 & 2 \\
\hline & & & 1,1 & 0,9 & & & 1,1 & 1 \\
\hline & $E T$ & 1 & 1,4 & 0,9 & NC & 1 & 1,7 & 1 \\
\hline & & 2 & 2,4 & 1,3 & & 2 & 2 & 1 \\
\hline & & & 1 & 0,4 & & & 0,3 & 0 \\
\hline & $\mathrm{SN}$ & 1 & 1,7 & 1,3 & & & & \\
\hline & & 2 & 1,9 & 1,6 & & & & \\
\hline & & & 0,2 & 0,3 & & & & \\
\hline CM1 & DY & 1 & 2,4 & 1,1 & CA & 1 & 2,5 & 1,5 \\
\hline & & 2 & 2,2 & 1 & & 2 & 2,7 & 1,8 \\
\hline & & & $-0,2$ & $-0,1$ & & & 0,2 & 0,3 \\
\hline & KY & 1 & 2,2 & 1 & $M X$ & 1 & 1,9 & 1,3 \\
\hline & & 2 & 2,9 & 1,4 & & 2 & 2,6 & 0,8 \\
\hline & & & 0,7 & 0,4 & & & 0,7 & $-0,5$ \\
\hline & $\mathrm{TE}$ & 1 & 2,7 & 1,9 & RA & 1 & 1,3 & 1,1 \\
\hline & & 2 & 2,9 & 1,5 & & 2 & 2,9 & 2,1 \\
\hline
\end{tabular}




\begin{tabular}{|l|l|l|c|c|c|c|c|c|}
\hline & & & 0,2 & $-\mathbf{0 , 4}$ & & & 1,6 & 1 \\
\hline $\mathrm{CM} 2$ & CE & 1 & 2,5 & 1,6 & $\mathrm{AX}$ & 1 & 2,1 & 2 \\
\hline & & 2 & 2,5 & 1,2 & & 2 & 2,7 & 2 \\
\hline & & & 0 & $-\mathbf{0 , 4}$ & & & 0,6 & 0 \\
\hline & $\mathrm{MO}$ & 1 & 2,6 & 2,6 & $\mathrm{GM}$ & 1 & 2,4 & 1,3 \\
\hline & & 2 & 2,8 & 2,4 & & 2 & 2,1 & 1,8 \\
\hline & & & 0,2 & $-\mathbf{0 , 2}$ & & $-\mathbf{0 , 3}$ & 0,5 \\
\hline
\end{tabular}

\begin{tabular}{|l|l|l|}
\hline Echelle & 3 compréhension immédiate & 3 réponses élaborées \\
\hline & 2 bonne compréhension & 2 réponses construites \\
\hline & 1 compréhension avec étayage & 1 réponses réflexes \\
\hline & 0 non compréhension & 0 sans réponse \\
\hline
\end{tabular}

Motivation pour des jeux spécifiques

Considérons maintenant l'effet de l'intervention sur la motivation pour des jeux spécifiques à l'ordinateur. Les questionnaires sur cette variable enquêtent non pas sur la motivation générale pour l'utilisation de l'ordinateur lors de séances d'anglais, mais plutôt sur le plaisir éprouvé par les participants à faire certaines activités en particulier. À la fin d'une période scolaire, les apprenants donnent leur avis sur les activités exploitées en anglais lors de cette période, les réponses étant fournies pendant l'atelier ordinateur du cours d'anglais. Les réponses de chaque participant sont présentées au tableau 5.

Tableau 5 - Appréciation des jeux spécifiques en ligne avant (1) et après (2) l'intervention.

\begin{tabular}{|l|l|l|l|l|l|l|l|l|l|l|}
\hline & \multicolumn{5}{|c|}{ TEST } & \multicolumn{5}{c|}{ CONTROLE } \\
\hline & & Game & Dictionary & & \multicolumn{2}{|c|}{ Game } & \multicolumn{2}{|c|}{ Dictionay } \\
\hline & & 1 & 2 & 1 & 2 & & 1 & 2 & 1 & 2 \\
\hline CE1 & DM & ++ & ++ & ++ & ++ & BP & + & + & ++ & - \\
\hline & & & & & & ST & ++ & - & - & + \\
\hline & & & & & & & & & & \\
\hline CE2 & EM & - & + & + & ++ & KT & - & + & ++ & ++ \\
\hline & ET & - & -- & ++ & -- & NC & ++ & + & ++ & $?$ \\
\hline
\end{tabular}




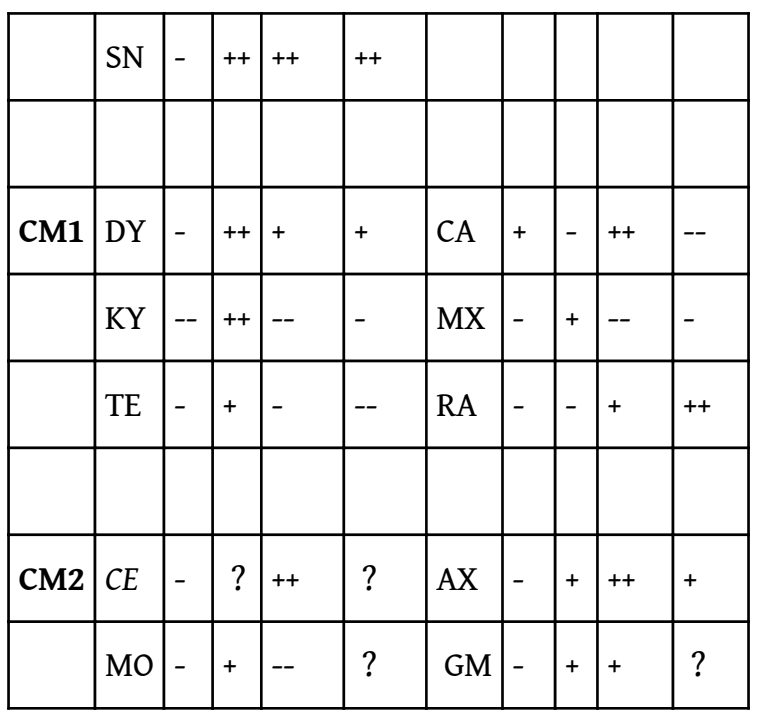

++ avis très positif

+ avis positif

- avis mitigé

- avis négatif

? impossible à interpréter

Il ressort de l'analyse de ces questionnaires que les apprenants du groupe test qui ont eu l'occasion pendant l'intervention de choisir librement entre les jeux proposés se retrouvent très majoritairement avec une évaluation très positive des jeux du site. À la différence du groupe cyberquête, sept apprenants sur neuf trouvent les jeux "bien" ou "très bien" (des deux autres, une apprenante ne les apprécie point, et pour l'autre nous n'avons pas de données). Seulement quatre apprenants du groupe contrôle terminent avec une opinion améliorée des jeux, et trois d'entre eux témoignent d'une motivation réduite. Ces résultats sont à comparer à l'opinion des apprenants d'un dictionnaire en ligne (languageguide.org), également exploité en cours; les avis sur ce dictionnaire ne progressent pas de la même façon.

\section{Temps passé à l'ordinateur en autonomie}

La dernière mesure de motivation de cette étude concerne le temps passé par chaque participant lors des activités de l'intervention. Cette mesure sert à vérifier l'activité des apprenants, et permet de rapprocher l'exposition à l'anglais d'éventuels progrès linguistiques. Les résultats présentés au tableau 6 démontrent premièrement une préférence pour les jeux interactifs, puisque les apprenants y ont consacré plus de 16 séances en moyenne, contre 13 pour la cyberquête. Parmi les cinq apprenants du groupe test qui passent le plus de temps (entre $2 \mathrm{~h} 50$ et $3 \mathrm{~h}$ ou plus) sur les activités, nous retrouvons les trois apprenants qui font preuve du plus de progrès en connaissances lexicales et interaction (DM, EM, et ET).

Tableau 6 - Temps passé à l'ordinateur en autonomie.

\begin{tabular}{|l|c|c|c|c|c|c|}
\hline Temps & \multicolumn{3}{|c|}{ TEST } & \multicolumn{3}{c|}{ CONTROLE } \\
\hline & & séances & minutes & & séances & minutes \\
\hline CE1 & DM & 18 & $3 \mathrm{~h}$ & BP & 19 & $3 \mathrm{~h} 10$ \\
\hline
\end{tabular}




\begin{tabular}{|c|c|c|c|c|c|c|}
\hline & & & & $S T$ & 19 & $3 \mathrm{~h} 10$ \\
\hline \multirow[t]{3}{*}{ CE2 } & EM & 17 & $2 \mathrm{~h} 50$ & $K T$ & 16 & $2 \mathrm{~h} 40$ \\
\hline & ET & 18 & $3 \mathrm{~h}$ & $\mathrm{NC}$ & 10 & $1 \mathrm{~h} 40$ \\
\hline & $\mathrm{SN}$ & 16 & $2 \mathrm{~h} 40$ & & & \\
\hline \multirow[t]{3}{*}{ CM1 } & DY & 16 & $2 \mathrm{~h} 40$ & $\mathrm{CA}$ & 13 & $2 \mathrm{~h} 10$ \\
\hline & KY & 16 & $2 \mathrm{~h} 40$ & $M X$ & 20 & $3 \mathrm{~h} 20$ \\
\hline & $\mathrm{TE}$ & 13 & $2 \mathrm{~h} 10$ & RA & 14 & $2 \mathrm{~h} 20$ \\
\hline \multirow[t]{2}{*}{ CM2 } & $C E$ & 20 & $3 \mathrm{~h} 20$ & $\mathrm{AX}$ & 16 & $2 \mathrm{~h} 40$ \\
\hline & MO & 17 & $2 \mathrm{~h} 50$ & GM & 15 & $2 \mathrm{~h} 30$ \\
\hline Moyenne & & 168 & & & 137 & \\
\hline
\end{tabular}

\section{Discussion}

L'expérience menée dans cette étude nous permet d'analyser les liens entre l'apprentissage de l'anglais médié par ordinateur à l'école primaire et différents aspects de la motivation pour apprendre du jeune apprenant. La méthode de recherche nous fournit l'occasion :

1. de comparer le niveau linguistique des apprenants avant et après un travail autonome sur des activités d'anglais en ligne ;

2. de contraster ces résultats avec ceux d'un groupe contrôle ;

3. de rapprocher les résultats linguistiques de mesures de motivation et d'autonomie.

Après analyse de mesures de reconnaissance lexicale et de capacité à interagir oralement avant et après l'intervention, nous constatons des améliorations légèrement plus marquées à la fois en quantité et en qualité chez les apprenants qui ont travaillé sur les activités en ligne. L'ensemble du groupe test avance (le score d'une apprenante restant stable) sur la mesure de reconnaissance lexicale, suggérant une amélioration du sentiment d'auto-efficacité en ce qui concerne le vocabulaire anglais, sinon un véritable progrès dans ce domaine. ${ }^{3}$ Une majorité du groupe fait preuve d'amélioration sur les deux mesures, montrant une capacité accrue à interagir à l'oral en même temps qu'une meilleure reconnaissance lexicale. Ce progrès est à déceler surtout parmi les apprenants les plus jeunes, qui ont le moins d'expérience de l'anglais. Nous remarquons un effet de plafond notamment dans les scores de compréhension pour les élèves de CM1 et CM2, et également en expression pour MO, où les scores avoisinent le maximum de 3. 

grande envergure et concernant moins d'apprenants. La majorité des apprenants ne font pas preuve de progrès dans les deux domaines de reconnaissance lexicale et interaction, et ceux pour lesquelles l'évolution est positive s'améliorent seulement sur l'une des deux mesures, pour la moitié d'entre eux. Nous ne pouvons pourtant pas parler d'effets marqués (ce qui n'étonnera guère, étant donné la courte durée de l'intervention), et nous restons très prudentes quant à l'interprétation de ces résultats, même s'ils restent encourageants pour des recherches ultérieures.

L'analyse des différentes mesures de motivation permet d'esquisser un certain nombre de profils d'apprenants qui peuvent en partie expliquer les résultats linguistiques déjà détaillés. Nous discernons, en premier lieu, un profil d'apprenant très motivé par l'utilisation de l'ordinateur. Cette motivation, indiquée par une préférence pour l'atelier ordinateur lors des séances d'anglais et souvent par une utilisation du site de la classe à la maison, semble être prioritaire sur tous les autres facteurs de motivation potentiels (enseignant, sentiment d'auto-efficacité). Les apprenants qui affichent cette motivation et qui ont pratiqué des activités d'anglais en ligne lors de l'intervention font preuve d'améliorations dans leur performance, et ce quelles que soient leurs orientations par ailleurs quant à l'enseignante ou à leur évaluation de leurs propres compétences en anglais.

61

Quatre apprenants du groupe contrôle présentent le même profil, et deux d'entre eux montrent une évolution linguistique positive sur l'une des deux mesures. KT l'explique ainsi, en réponse à la question: Penses-tu que tu arrives bien en cours d'anglais? Pourquoi ? Oui, parce que j'en fais à la maison. Nos travaux permettent également de dégager un effet positif sur la motivation pour la pratique de jeux en ligne, par opposition à la cyberquête : les apprenants ayant pratiqué les jeux en autonomie affichent une opinion plus positive de ceux-ci par rapport à d'autres activités en ligne (dictionnaire) et par rapport aux apprenants ayant pratiqué la cyberquête.

62 Le deuxième profil apprenant qui se dégage de ces travaux concerne l'apprenant attaché à l'enseignant et manquant de confiance en ses capacités en anglais. C'est le cas de quatre participants dans l'étude, dont trois sont parmi les plus jeunes, âgés de 7 à 8 ans. Nos résultats confirment sur ce point ceux de Nikolov (1999), qui note un attachement à l'enseignant plus marqué chez les élèves les plus jeunes (les 6 à 8 ans dans son cas), ainsi que les travaux de Burstall (1980) qui relève une meilleure relation enseignant-élève dans les petites écoles rurales.

63

Enfin, nous dégageons un troisième profil d'apprenant qui semble plafonner en cours d'anglais. Cet effet de plafond peut s'expliquer en partie par des tests trop faciles (car prévus pour une classe à quatre niveaux et surtout pour éviter l'impression d'échec chez une population fragile, cf. Hasselgreen, 2000). Mais il paraît également possible que l'apprenant qui juge sa maitrise de l'anglais suffisante selon les normes de la classe ne fournisse pas les efforts nécessaires pour faire des progrès supplémentaires. Cette analyse nous rapproche des notions, d'une part, de besoin d'accomplissement de l'apprenant, et, d'autre part, des normes de la situation de la classe dans le modèle de Dörnyei et Csizér $(1998,2005)$. Notre résultat diffère de celui de Mills et al. (2007), qui trouve une relation positive entre sentiment d'efficacité personnelle et note de fin de semestre pour des débutants adultes de français langue étrangère. 

cette étude, nous pourrons en tirer les conclusions suivantes en ce qui concerne les relations entre la motivation et le potentiel pour l'apprentissage de l'anglais à l'école médié par l'ordinateur. En premier lieu, il semble que les apprenants qui affichent une préférence nette pour le travail à l'ordinateur sont en position favorisée: leur appréciation de ce mode de travail leur permet de tirer profit des jeux d'anglais et les pousse même à chercher des occasions supplémentaires de les pratiquer en dehors du temps scolaire. Ensuite, les apprenants qui cherchent à faire plaisir à l'enseignant et qui estiment avoir des difficultés en anglais semblent également se trouver dans une situation favorable, avec une motivation qui leur permet de bénéficier du travail qui leur est demandé en classe et en autonomie. Enfin, les apprenants qui ont un sentiment d'efficacité personnelle robuste quant au cours d'anglais ne progressent pas; sans motivation spécifique pour l'ordinateur ou sentiment d'un retard à combler en anglais, ils ne profitent pas du temps passé aux jeux en ligne.

\section{Conclusion}

Si cette étude démontre la faisabilité et l'utilité d'un travail autonome sur des activités d'anglais en ligne pour le jeune apprenant, elle souligne aussi la multiplicité des motivations qui peuvent influer sur ses chances de progresser.

Le lecteur ne sera pas étonné de voir apparaître dans ce travail un lien entre l'intérêt de l'apprenant pour le travail à l'ordinateur de manière générale et sa capacité à tirer un profit linguistique des jeux d'anglais en ligne. Dans leurs travaux sur l'apprentissage des TICE par le jeu (through play), Kennewell et Morgan (2006) notent une corrélation entre le sentiment d'efficacité personnelle pour l'utilisation d'un ordinateur et pour les jeux vidéo d'une part, et les compétences TICE acquises lors de l'intervention de l'autre. Les apprenants qui se considèrent comme utilisateurs efficaces de l'outil informatique réagissent mieux en situation d'apprentissage d'un nouveau logiciel.

La relation négative établie par la présente étude entre le sentiment d'efficacité personnelle pour l'apprentissage de l'anglais et les mesures linguistiques de lexique et d'interaction est plus surprenante. La plupart des recherches précédentes font état d'une corrélation positive (voir par exemple Ehrman et al., 2003), mais dans nos travaux, nous trouvons des apprenants qui ont un sentiment faible d'efficacité personnelle mais qui progressent, et d'autres avec un sentiment fort d'efficacité personnelle qui ne progressent pas. À ce jour, nous ne connaissons pas d'étude empirique qui confronterait le niveau linguistique avec la motivation chez le jeune apprenant ; il serait intéressant de travailler dans ce sens pour creuser les profils esquissés ici.

68

Enfin le dernier résultat inattendu de cette étude concerne la maturité des apprenants qui font le plus de progrès. Contrairement à ce que l'on pourrait imaginer, ce ne sont pas les élèves les plus âgés ni ceux que les enseignants jugent être les plus autonomes qui profitent le plus de l'apprentissage médié par ordinateur. Les progrès les plus marqués concernent les élèves les plus jeunes. Tout porte à croire qu'une approche constructiviste qui laisse la liberté à l'apprenant quant aux choix des ressources et des stratégies d'apprentissage peut porter ses fruits dès les débuts de l'apprentissage en langue étrangère... 
69 convient néanmoins d'apporter quelques mises en garde quant à la possible généralisation des résultats de cette étude. Il s'agit évidemment d'une petite expérience menée sur une très courte durée avec peu de participants et qui donne ainsi des résultats mitigés, qui sont pourtant de bonne augure pour des travaux ultérieurs. Des recherches futures avec un panel d'apprenants plus large et des instruments de mesures linguistiques et motivationnelles plus précis permettraient d'affiner les conclusions présentées ici.

Il est également important de remarquer que, même si les progrès observés dans cette étude doivent provenir d'un travail en autonomie en dehors du temps du cours d'anglais (puisqu'ils sont plus marqués chez les apprenants ayant pratiqué des jeux d'anglais que chez ceux qui ont effectué des cyberquêtes en français), ce travail en autonomie s'est pourtant poursuivi en parallèle avec des séances d'anglais axées fortement sur l'exploitation du même type de ressources en ligne. La situation d'apprentissage inclut de cette manière la présentation de stratégies d'apprentissage qui sont susceptibles de conditionner le comportement des apprenants face aux jeux par la suite. Il ne s'agit donc nullement d'inciter à l'abandon de méthodes d'enseignement de langue étrangère traditionnelles au profit de séances de jeux d'anglais libres. En revanche, nous estimons avoir apporté un début de preuve de l'utilité de jeux en ligne en complément de séances d'anglais habituelles, et qui nous conforte dans notre volonté d'étudier les relations complexes entre la motivation, l'autonomie, le jeu, et l'acquisition de langues étrangères chez le jeune apprenant.

\section{BIBLIOGRAPHIE}

Bandura, A. (2001). "Social cognitive theory : an agentic perspective". Annual Review of Psychology. vol. 52. pp. 1-26.

Bertin, J.- C. \& Narcy-Combes, J.-P. (2007). "Monitoring the learner - who, why and what for?". Computer Assisted Language Learning, vol. 20, nº 5. pp. 443-457.

Blin, F. (2004). "CALL and the development of learner autonomy". ReCALL, vol. 16, nº 2. pp. 377-395.

Burstall, C. (1980). "Primary French in the balance." In Holden, S. (dir.). Teaching children. Modern English Publications : London.

Catroux, M. (2006). "L'apprentissage collaboratif médiatisé par Internet : conditions de mise en oeuvre chez de jeunes apprenants d'anglais". Les Cahiers de l'Acedle, oㅡ․ pp. 52-73.

Chapelle, C. (1998). "Multimedia CALL: Lessons to be learned from research on instructed SLA". Language Learning and Technology, vol. 2, no 1. pp. 21-39. Disponible en ligne. http://llt.msu.edu/ vol2num1/chapelle/

Cotterall, S. (1999). "Key variables in language learning: what do learners believe about them?". System, vol. 27. pp. 493-531. 
Dörnyei, Z. \& Csizér, K. (1998). "Ten commandments for motivating language learners: results of an empirical study." Language Teaching Research, vol. 2, nº 3. pp. 203-229.

Dörnyei, Z. \& Csizér, K. (2005). "Language learners motivational profiles and their motivated learning behaviour." Language Learning, vol. 55, no 4. pp. 613-659.

Ehrman, M., Leaver, B. \& Oxford, R. (2003). "A brief overview of individual differences in second language learning". System, vol. 31. pp. 313-330.

Felix, U. (2005). "E-learning in the third millenium: the need for combining social and cognitive constructivist approaches". ReCALL, vol. 17, no 1. pp. 85-100.

Figura, K. \& Jarvis, H. (2007). "Computer-based materials: A study of learner autonomy and strategies". System, vol. 35, pp. 448-468.

Firth, A. \& Wagner, J. (1997). On discourse, communication, and (some) fundamental concepts in SLA research. The Modern Language Journal, vol. 81. pp. 285-300. Ré-édité en 2007 dans The Modern Language Journal, vol. 91. pp. 757-772.

Gardner, R. (1985). Social psychology and second language learning. London: Edward Arnold.

Gardner, R. \& Lambert, W. (1972). Attitudes and motivation in second language learning. Rowley, MA, USA : Newbury House.

Grosbois, M. (2007). "Didactique des langues et recherche expérimentale". Les Cahiers de l'Acedle, no 4 . pp. 65-83.

Hasselgreen, A. (2000). "The assessment of the English ability of young learners in Norwegian schools: an innovative approach". Language Testing, vol. 17, nº 2. pp. 261-77.

Johnson, M. (2004). A philosophy of second language acquisition. New Haven, CT: Yale University Press.

Jonassen, D., Davidson, M., Collins, M., Campbell, J. \& Bannan Haag, B. (1995). "Constructivism and computer-mediated communication in distance education". American Journal of Distance Education, vol. 9, nº 2. pp. 7-25.

Jones, L. (2004). "Testing L2 vocabulary recognition and recall using pictorial and written tests". Language Learning and Technology, vol. 8, no 3. pp. 122-143. http://llt.msu.edu/vol8num3/jones/.

Kennewell, S. \& Morgan, A. (2006). "Factors influencing learning through play in ICT settings". Computers and Education, vol. 46. pp. 265-279.

Liem, A. D., Lau, S. \& Nie, Y. (à paraître). "The role of self-efficacy, task value, and achievement goals in predicting learning strategies, task disengagement, peer relationship, and achievement outcome." Contemporary Educational Psychology.

Manolopoulou-Sergi, E. (2004). "Motivation within the information processing model of foreign language learning". System, vol. 32, pp. 427-441.

Mills, N., Pajares, F. \& Herron, C. (2007). "Self-efficacy of College Intermediate French Students: Relation to Achievement and Motivation". Language Learning, vol. 57, no 3. pp. 417-442.

Nikolov, M. (1999). "'Why do you learn English? Because the teacher is short.' A study of Hungarian children's foreign language learning motivation". Language Teaching Research, vol. 3, no 1. pp. 33-56.

Ramirez Verdugo, D. \& Alonso Belmonte, I. (2007). "Using digital stories to improve listening comprehension with Spanish young learners of English". Language Learning and Technology, vol. 11, no 1. pp. 87-101. http://llt.msu.edu/vol11num1/ramirez/. 
Read, J., MacFarlane, S. \& Casey, C. (2002). "Endurability, Engagement and Expectations: Measuring children's fun". http://www.chici.org/references/endurability_engagement.pdf. Sieloff-Magnan, S. (2007). Presenting the Focus Issue. The Modern Language Journal, vol. 91. pp. 733-734.

Simina, V. \& Hamel, M.-J. (2005). "CASLA through a social constructivist perspective: WebQuest in project-driven language learning." ReCALL, vol. 17, no 2. pp. 217-228.

Spratt, M, Humphreys, G. \& Chan, V. (2002). "Autonomy and motivation: which comes first?". Language Teaching Research, vol. 6, no 3. pp. 245-266.

Wigfield, A. \& Eccles, J. (2000). "Expectancy-value theory of achievement motivation". Contemporary Educational Psychology, vol. 25. pp. 68-81.

\section{Sites Internet}

Site expérimental Cherry English. Consulté le 5 novembre 2008 : http://www.unice.fr/whyte/ teach/early/cherry/index.html.

Kindersite project. Consulté le 5 novembre 2008 : http://www.kindersite.org/.

Languageguide. Consulté le 5 novembre 2008 : http://languageguide.org/.

\section{ANNEXES}

Annexe 1 : Codage de la compréhension et de l'expression des apprenants

\begin{tabular}{|c|c|c|}
\hline \multicolumn{3}{|c|}{ Compréhension } \\
\hline Score & Descriptif & Exemple \\
\hline 3 & $\begin{array}{l}\text { réponse immédiate (question } \\
\text { posée une seule fois ou avec } \\
\text { répétition immédiate des } \\
\text { éléments essentiels) }\end{array}$ & $\begin{array}{l}\text { SW What colour are your scissors? } \\
\text { CA Uh red uh orange. }\end{array}$ \\
\hline 2 & $\begin{array}{l}\text { réponse avec aide verbale } \\
\text { (reformulation de la question } \\
\text { et répétitions appuyées des } \\
\text { éléments clés) }\end{array}$ & $\begin{array}{l}\text { SW What's your name? } \\
\text { BP Blue SW No, no. Your name, name. } \\
\text { What's your name? BP BP. } \\
\text { SW BP, OK that's good. }\end{array}$ \\
\hline 1 & $\begin{array}{l}\text { réponse à l'aide d'images } \\
\text { (indication de l'image } \\
\text { pertinente), ou réponse 'à } \\
\text { côté' }\end{array}$ & $\begin{array}{l}\text { SW Do you know what the month is? The month? } \\
\text { January, February? March? } \\
\text { NC October } \\
\text { SW No, no, now. Oh, your birthday. OK now, now not } \\
\text { January, not February }\end{array}$ \\
\hline
\end{tabular}




\begin{tabular}{|l|l|l|}
\hline 0 & $\begin{array}{l}\text { absence de réponse } \\
\text { (abandon par l'interlocuteur), } \\
\text { traduction par l'interlocuteur, ou } \\
\text { réponse indiquant un } \\
\text { manque de compréhension }\end{array}$ & $\begin{array}{l}\text { SW Show me some fruit. Fruit. } \\
\text { DY Oh là. } \\
\text { SW No that's not fruit. We eat fruit. We eat fruit. } \\
\text { Doesn't matter. }\end{array}$ \\
\hline
\end{tabular}

\begin{tabular}{|c|c|c|}
\hline \multicolumn{3}{|c|}{ Expression } \\
\hline Score & Descriptif & Exemple \\
\hline 3 & $\begin{array}{l}\text { Réponse en deux mots ou } \\
\text { plus (phrase, expression, } \\
\text { liste) }\end{array}$ & $\begin{array}{l}\text { SW What's the weather today? } \\
\text { CA The weather is sunny. }\end{array}$ \\
\hline 2 & $\begin{array}{l}\text { Réponse d'un seul (nouveau) } \\
\text { mot (exceptés : répétition de } \\
\text { la question, yes/no, son } \\
\text { prénom) }\end{array}$ & $\begin{array}{l}\text { SW Have you got a cat? } \\
\text { EM Three } \\
\text { SW Three cats. Goodness. }\end{array}$ \\
\hline 1 & $\begin{array}{l}\text { Réponse par Yes ou No } \\
\text { (oui/non) ou par écho de } \\
\text { l'interlocteur }\end{array}$ & $\begin{array}{l}\text { SW Have you got a rabbit? } \\
\text { ET No. }\end{array}$ \\
\hline 0 & $\begin{array}{l}\text { Absence de réponse, réponse } \\
\text { en français, réponse non- } \\
\text { verbale }\end{array}$ & L'élève montre une image avec son doigt. \\
\hline
\end{tabular}

Annexe 2 : Questionnaire sur la motivation générale pour apprendre l'anglais

1. Est-ce que tu aimes apprendre l'anglais?

2. À ton avis, pourquoi apprend-on l'anglais à l'école?

3. Penses-tu que tu arrives bien en cours d'anglais ? Pourquoi ?

4. L'anglais est une matière difficile - vrai ou faux ? Pourquoi ?

5. Quand on travaille en groupe, qu'est-ce que tu préfères - l'ordinateur, le travail en autonomie, ou avec Shona?

\section{NOTES}

1. Cette façon de concevoir l'expérience permet d'examiner l'effet d'un travail supplémentaire par certains élèves sur des activités en anglais, supposées aider l'acquisition, tout en respectant les exigences pédagogiques de la séance d'anglais, en proposant aux autres élèves un travail sur la culture des pays anglophones mais qui n'expose pas à la langue anglaise.

2. Nous comprenons que cet apprenant a observé une tendance chez son enseignante à interroger d'abord et surtout les élèves qu'elle considère comme étant plus faibles ; remarquant 
qu'elle ne l'interroge que rarement, MO en conclut qu'il fait partie des meilleurs élèves en anglais.

3. Il était prévu d'inclure aux travaux une mesure de production orale en demandant aux apprenants de nommer les images lors de l'entretien oral, mais des difficultés d'exécution et d'analyse ont conduit à son abandon. Lors du pré-test, l'auteur a constaté une certaine frustration chez les élèves lorsque le temps alloué pour l'entretien ne leur permettait pas d'énumérer tous les mots qu'ils croyaient connaître. Au post-test, nous leur avons permis de nommer toutes les images choisies, avec l'intention de comparer les scores par une mesure de vitesse d'énumération (mots par seconde). Des problèmes liés aux conditions d'enregistrement (dans la classe) ont rendu cette mesure difficilement exploitable et donc peu fiable.

\section{RÉSUMÉS}

Cette étude empirique de l'effet des activités médiées par ordinateur pour la motivation en apprentissage de l'anglais langue étrangère à l'école primaire en France s'inspire de l'offre grandissante de jeux en ligne conçus à cet effet. Les participants sont dix-huit élèves d'une classe multi-niveaux qui ont travaillé en autonomie à l'ordinateur ; nous mesurons leur reconnaissance lexicale, leur capacité à interagir oralement et leurs motivations avant et après l'intervention. Nous comparons ensuite les performances d'un groupe test travaillant sur des activités d'anglais en ligne avec celles d'un groupe de contrôle travaillant sur une cyberquête en français. Nous notons des performances supérieures pour le groupe test sur quelques mesures et pour certains types de motivation, notamment pour les apprenants avec un goût prononcé pour le travail à l'ordinateur et pour ceux qui sont attachés à l'enseignant mais qui ont un faible sentiment d'efficacité personnelle pour l'anglais. Ces résultats nous encouragent à poursuivre l'étude de l'utilité des jeux en ligne pour l'apprentissage de l'anglais langue étrangère ainsi que l'étude de la relation entre motivation et apprentissage des langues à l'école primaire.

The widespread availability of web-based games for learning English provided the impetus for this empirical study of the effectiveness of such activities for motivation and acquisition in young learners in a French primary school context. Eighteen pupils aged 7 to 11 in an experimental EFL class participated in a study of autonomous learning by computer, using measures of lexical recognition, oral interaction, and motivation. The group working on web-based EFL games outperformed a control group using webquests in French on some measures, with the best results obtained by learners with special motivation for computers and those with low selfefficacy for learning English but strong affiliation for the teacher. The results support further research into the utility of web-based games in the EFL classroom and the relationship between motivation and learning in the primary second language classroom.

\section{INDEX}

Mots-clés : ALAO, jeune apprenant, motivation, autonomie

Keywords : CALL, young learner, motivation, autonomy 


\section{AUTEUR}

\section{SHONA WHYTE}

Shona Whyte est maître de conférences à l'université de Nice-Sophia Antipolis. Elle s'intéresse à l'acquisition des langues secondes en particulier chez le jeune apprenant ainsi qu'à l'apport des nouvelles technologies à l'enseignement et à l'apprentissage.

Courriel : whyte[at]unice.fr.

Toile : http://www.unice.fr/whyte.

Adresse: Faculté des lettres, arts et sciences humaines, université de Nice-Sophia Antipolis, 98 boulevard Herriot, BP 3209, 06204 Nice Cedex 3, France. 